EPJ Web of Conferences 116, 03005 (2016)

DOI: $10.1051 /$ epjconf/201611603005

(C) Owned by the authors, published by EDP Sciences, 2016

\title{
The Giant Radio Array for Neutrino Detection
}

Olivier Martineau-Huynh ${ }^{1}$, Kumiko Kotera ${ }^{2}$, Mauricio Bustamente ${ }^{3,4}$, Didier Charrier ${ }^{5}$, Sijbrand De Jong $^{6}$, Krijn D. de Vries ${ }^{7}$, Ke Fang ${ }^{8}$, Zhaoyang Feng ${ }^{9}$, Chad Finley ${ }^{10}$, Quanbu Gou ${ }^{9}$, Junhua Gu ${ }^{10}$, Jordan C. Hanson ${ }^{3,4}$, Hongbo $\mathrm{Hu}^{9}$, Kohta Murase ${ }^{12}$, Valentin Niess ${ }^{13}$, Foteini Oikonomou ${ }^{12}$, Nicolas Renault-Tinacci ${ }^{4}$, Julia Schmid ${ }^{14}$, Charles Timmermans ${ }^{5}$, Zhen Wang ${ }^{9}$, Xiangping Wu ${ }^{11}$, Jianli Zhang ${ }^{11}$, and Yi Zhang ${ }^{9}$

${ }^{1}$ LPNHE, CNRS-IN2P3 and Universités Paris VI \& VII, 4 place Jussieu, 75252 Paris, France

${ }^{2}$ Institut d'Astrophysique de Paris, Sorbonne Universités, UPMC Univ. Paris 6 and CNRS, UMR 7095, 98 bis bd Arago, 75014 Paris, France

${ }^{3}$ Center for Cosmology and AstroParticle Physics, The Ohio State University, Columbus, OH 43210 , USA

${ }^{4}$ Department of Physics, The Ohio State University, Columbus, OH 43210, USA

${ }^{5}$ SUBATECH, IN2P3-CNRS, Université de Nantes, Ecole des Mines de Nantes, Nantes, France

${ }^{6}$ Nikhef/Radboud University, Nijmegen, The Netherlands

${ }^{7}$ Vrije Universiteit Brussel, Dienst ELEM, 1050 Brussels, Belgium

${ }^{8}$ Department of Astronomy and Astrophysics, University of Chicago, Chicago, IL 60637, USA

${ }^{9}$ Key Laboratory of Particle Astrophysics, Institute of High Energy Physics, Chinese Academy of Sciences, Beijing 100049, China

${ }^{10}$ Oskar Klein Centre and Dept. of Physics, Stockholm University, 10691 Stockholm, Sweden

${ }^{11}$ National Astronomical Observatory, Chinese Academy of Sciences, Beijing 100012, China

${ }^{12}$ Department of Physics, Department of Astronomy \& Astrophysics, Pennsylvania State University, University Park, PA, USA

${ }^{13}$ Clermont Université, Université Blaise Pascal, CNRS/IN2P3, Laboratoire de Physique Corpusculaire, BP. 10448, 63000 Clermond-Ferrand, France

${ }^{14}$ Laboratoire AIM, Université Paris Diderot/CEA-IRFU/CNRS, Service d'Astrophysique, CEA Saclay, 91191 Gif-sur-Yvette, France

\begin{abstract}
High-energy neutrino astronomy will probe the working of the most violent phenomena in the Universe. The Giant Radio Array for Neutrino Detection (GRAND) project consists of an array of $\sim 10^{5}$ radio antennas deployed over $\sim 200000 \mathrm{~km}^{2}$ in a mountainous site. It aims at detecting high-energy neutrinos via the measurement of air showers induced by the decay in the atmosphere of $\tau$ leptons produced by the interaction of cosmic neutrinos under the Earth surface. Our objective with GRAND is to reach a neutrino sensitivity of $5 \times 10^{-11} E^{-2} \mathrm{GeV}^{-1} \mathrm{~cm}^{-2} \mathrm{~s}^{-1} \mathrm{sr}^{-1}$ above $3 \times 10^{16} \mathrm{eV}$. This sensitivity ensures the detection of cosmogenic neutrinos in the most pessimistic source models, and up to 100 events per year are expected for the standard models. GRAND would also probe the neutrino signals produced at the potential sources of UHECRs.
\end{abstract}

This is an Open Access article distributed under the terms of the Creative Commons Attribution License 4.0, which permits unrestricted use, distribution, and reproduction in any medium, provided the original work is properly cited. 


\section{Detection method}

Cosmic $v_{\tau} \mathrm{s}$ can produce $\tau$ particles underground through charged current interaction. $\tau \mathrm{s}$ travel to the surface of the Earth and decay in the atmosphere, generating Earth-skimming extensive air showers $(\text { EAS })^{1}[1,2]$. Coherent electromagnetic radiation is associated to the shower development at frequencies of a few to hundreds of $\mathrm{MHz}$ at a detectable level for showers with $E \gtrsim 10^{17} \mathrm{eV}$.

The strong beaming of the electromagnetic emission combined with the transparency of the atmosphere to radio waves will allow the radio-detection of EAS initiated by $\tau$ decays at distances up to several tens of kilometers. Radio antennas are thus ideal instruments for this purpose. Furthermore, they offer practical advantages (limited unit cost, easiness of deployment, ...) that allow the deployment of an array over very large areas, as required by the expected low neutrino rate.

Remote sites, with low electromagnetic background, should obviously be considered for the array location. In addition, mountain ranges are preferred, first because they offer an additional target for the neutrinos, and also because mountain slopes are better suited to the detection of horizontal showers compared to flat areas, parallel to the showers trajectories.

GRAND antennas are foreseen to operate in the $30-100 \mathrm{MHz}$ frequency band. An extension of the antenna bandwidth up to 200 or $300 \mathrm{MHz}$ would enable to observe the Cherenkov ring associated with the air shower, that represents a sizable fraction of the total electromagnetic signal and may provide an unambiguous signature for background rejection.

\section{GRAND layout and neutrino sensitivity}

We present here a preliminary evaluation of the potential of GRAND for the detection of cosmic neutrinos. We initially simulated the response of a setup of 40000 antennas deployed on a square layout of $60000 \mathrm{~km}^{2}$ in a remote mountainous area (Tianshan mountains, XinJiang, China).

Simulation method. We perform a 1D tracking of a primary $v_{\tau}$, simulated down to the converted $\tau$ decay. Standard rock with a density of $2.65 \mathrm{~g} / \mathrm{cm}^{3}$ is assumed down to sea level and the Earth core is modeled with the Preliminary Reference Earth Model [3]. The simulation of the Deep Inelastic Scattering of the neutrinos is performed with Pythia6.4, using the CTEQ5d probability distribution functions (PDF) combined with [4] for cross section calculations. The propagation of the produced $\tau$ is simulated using randomized values from parameterizations of GEANT4.9 PDFs for $\tau$ path length and proper time. Photonuclear interactions in GEANT4.9 have been extended above PeV energies following [5]. The $\tau$ decay is simulated using the TAUOLA package.

For the time being only an analytical treatment of EAS radio detection has been performed. ZHAireS simulations [6] of horizontal showers have been used to parametrize the distance at which radio antennas may detect EAS radio emissions. Assuming an aggressive (conservative) detection threshold $\epsilon_{a}=30 \mu \mathrm{V} / \mathrm{m}\left(\epsilon_{c}=100 \mu \mathrm{V} / \mathrm{m}\right)$, it was found that a conical detection volume centered on the shower axis could be considered, with a half-angle between $1.4^{\circ}\left(0.9^{\circ}\right)$ for a shower energy $E_{s h}=10^{17} \mathrm{eV}$ and $4.7^{\circ}\left(3.8^{\circ}\right)$ for $E_{s h}=3 \times 10^{20} \mathrm{eV}$ and a distance to the $\tau$ decay point between 14 and $90 \mathrm{~km}(45 \mathrm{~km})$ for $E_{s h}=10^{17} \mathrm{eV}$, and between 14 and $300 \mathrm{~km}(200 \mathrm{~km})$ for $E_{s h}=3 \times 10^{20} \mathrm{eV}$. We considered that a shower is detected if a cluster of 8 neighbouring antennas or more is inside this detection volume and in direct view of the $\tau$ decay point.

Note that the Cherenkov effect is not taken into account in this study. This will be done in the up-coming full Monte-Carlo study of the GRAND neutrino sensitivity.

\footnotetext{
${ }^{1}$ Other neutrino flavors can be neglected as the electron range in matter at these energies is too short and the muon decay length too large compared to flight distances underground.
} 

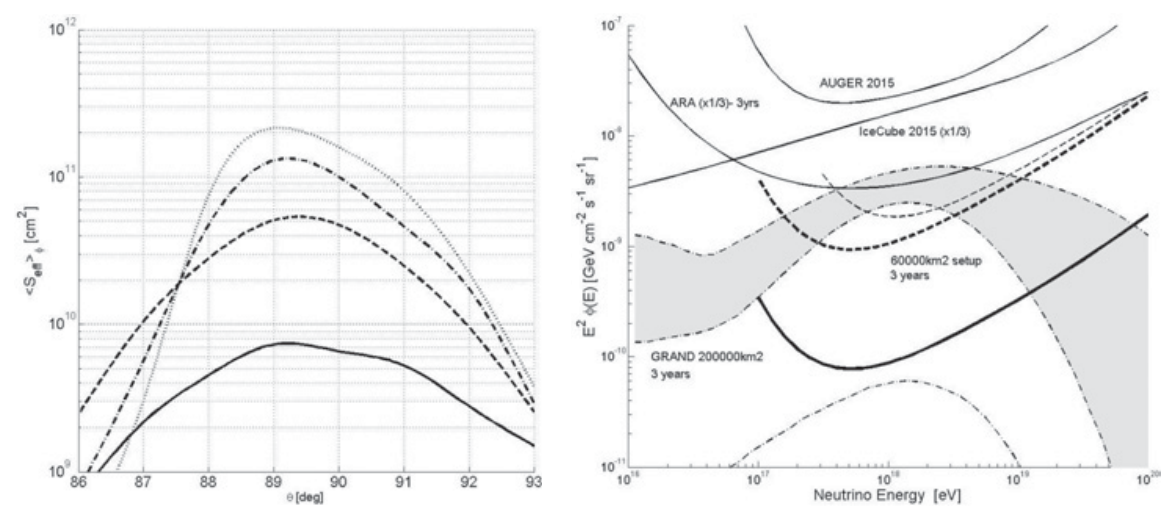

Figure 1. Left: effective area of the $60000 \mathrm{~km}^{2}$ simulated set-up averaged over azimuth angle $\phi$ as a function of zenith angle $\theta$ for various initial $v_{\tau}$ energies (plain line: $10^{17.5} \mathrm{eV}$, dashed: $10^{18.5} \mathrm{eV}$, dash-dotted: $10^{19.5} \mathrm{eV}$, dotted: $10^{20.5} \mathrm{eV}$ ). Zenith angles $>90^{\circ}$ correspond here to down-going trajectories. Right: differential sensitivity limit of the $60000 \mathrm{~km}^{2}$ simulated setup (dashed line, thin: conservative, thick: aggressive), of the projected 3 times larger GRAND array (thick line), and for other instruments (thin lines), and estimated theoretical cosmogenic neutrino fluxes for one neutrino flavor [8].

Results and implications. Assuming a 3-year observation on an area of $60000 \mathrm{~km}^{2}$ with no neutrino candidate, a $90 \%$ C.L. integral limit of $6.6 \times 10^{-10}\left(1.3 \times 10^{-9}\right) \mathrm{GeV}^{-1} \mathrm{~cm}^{-2} \mathrm{~s}^{-1}$ can be derived for an $E^{-2}$ neutrino flux in our aggressive (conservative) scenario.

This preliminary analysis also demonstrates that mountains constitute a sizable target for neutrinos, with $\sim 50 \%$ of down-going events, corresponding to neutrinos interacting inside the mountains (Fig. 1). It also appears that specific parts of the array (large mountains slopes facing another mountain range at distances of 30-80 km) are associated with a detection rate well above the average. We thus realized that a factor of 10 improvement in sensitivity - corresponding to cosmogenic neutrinos event rates between 1 to 100 per year - may be reached with a factor 3 increase in the detector area only, provided the detector is composed of several sub-arrays of smaller size (few $10000 \mathrm{~km}^{2}$ ) deployed solely on favorable sites. This is the envisioned GRAND setup.

The expected angular resolution on the arrival direction of the events detected was computed analytically following [7]. A mean resolution of $0.05^{\circ}$ should be achievable for a 3 ns precision on the antenna trigger timing. Energy estimation will be challenging, mainly because the $\tau$ decay point is unknown. The very specific ground pattern of the electric field amplitude and polarization associated with $v$-induced EAS should provide a very efficient tool for the discrimination of background (very inclined EAS induced by UHECRs or events of anthropic origin for example). This shall be tested on an engineering array of size $\sim 1000 \mathrm{~km}^{2}$. This array will obviously be too small to perform a neutrino search, but cosmic rays should be detected above $10^{18} \mathrm{eV}$. Their reconstructed properties will enable us to validate this stage, if found to be compatible with expectations.

\section{High-energy neutrino astronomy with GRAND and other science cases}

UHECRs are likely produced in extragalactic sources, given the strength of Galactic magnetic fields and the lack of correlations with the Galactic plane. Some fraction of their energy is converted to highenergy neutrinos through the decay of charged pions produced by interactions with ambient matter and radiation. This can happen in the source environment or during the flight in the intergalactic medium (cosmogenic neutrinos). The range of expected cosmogenic neutrino fluxes can be calculated precisely, 
and depends mostly on parameters inherent to the cosmic rays (Fig. 1) [8]. The calculated level of highenergy neutrino fluxes produced at the sources depends on the modeling of the acceleration region, but robust flux estimates have recently been derived for some sources [9-12], arguing that many scenarios can be tested with increased sensitivities.

The sensitivity of GRAND should guarantee the detection of cosmogenic EeV neutrinos. For reasonable source scenarios (Fig. 1), GRAND aims at collecting up to 100 events per year above $10^{17} \mathrm{eV}$. The ideal way to identify high-energy neutrino sources would be to observe a point source - a transient source is a likely candidate due to the power and density constraints from UHECR observations (e.g., [13]). GRAND opens this possibility with its excellent spatial resolution.

Given its effective area, GRAND would also be the largest UHECR observatory on ground, and should provide valuable information to help resolve these puzzles if precision of reconstructed parameters is satisfactory. Determination of the UHECR composition in particular is a key issue. Detailed simulation studies will be performed to estimate performances reachable by GRAND.

The large array size and the frequency range of GRAND - if extended to $300 \mathrm{MHz}$ - could be profitable for other physics goals (such as the study of the Epoch of Reionization [14] or the search for Fast Radio Bursts [15]). We are presently assessing the technical cost of pushing the detector towards these directions.

\section{Conclusion}

The GRAND project aims at building a next-generation neutrino telescope composed of radio antennas deployed over $\sim 200000 \mathrm{~km}^{2}$. Preliminary simulations indicate that a sensitivity guaranteeing the detection of cosmogenic neutrinos may be achievable. GRAND would also be a powerful instrument for UHECR detection at energy $\gtrsim 10^{19} \mathrm{eV}$. Work is ongoing to determine precisely the achievable scientific goals and the corresponding technical constraints.

We thank J. Alvarez-Muniz and W. Carvalho for helpful comments and discussions. The GRAND and GRANDproto projects are supported by the Institut Lagrange de Paris, the France China Particle Physics Laboratory, the Natural Science Fundation of China (Nos.11135010, 11375209) and the Chinese Ministery of Science and Technology.

\section{References}

[1] D. Fargion, International Cosmic Ray Conference 2, 396 (1999), astro-ph/9906450

[2] X. Bertou, et al, Astroparticle Physics 17, 183 (2002), astro-ph/0104452

[3] A. Dziewonski, D. Anderson, Physics of the Earth and Planetary Interiors 25(4), 297 (1981)

[4] R. Gandhi, C. Quigg, M.H. Reno, I. Sarcevic, Phys. Rev. D 58(9), 093009 (1998), hep-ph/9807264

[5] S. Dutta, et al., European Physical Journal C 18, 405 (2000), hep-ph/9905475

[6] J. Alvarez-Muniz, W.R. Carvalho, Jr., E. Zas, Astropart. Phys. 35, 325 (2012), 1107.1189

[7] D. Ardouin et al., Astroparticle Physics 34, 717 (2011), 1007. 4359

[8] K. Kotera, D. Allard, A.V. Olinto, J. Cos. and Astro. Phys. 10, 13 (2010), 1009. 1382

[9] P. Baerwald, M. Bustamante, W. Winter, Astroparticle Physics 62, 66 (2015), 1401.1820

[10] K. Murase, P. Mészáros, B. Zhang, Phys. Rev. D 79(10), 103001 (2009), 0904.2509

[11] K. Fang, K. Kotera, K. Murase, A.V. Olinto, Phys. Rev. D 90(10), 103005 (2014)

[12] K. Murase, Y. Inoue, C.D. Dermer, Phys. Rev. D 90(2), 023007 (2014), 1403.4089

[13] K. Murase, H. Takami, ApJ Letters 690, L14 (2009), 0810.1813 
Very Large Volume Neutrino Telescope (VLVnT-2015)

[14] S. Zaroubi, The Epoch of Reionization, in Astrophysics and Space Science Library, edited by T. Wiklind, B. Mobasher, V. Bromm (2013), Vol. 396 of Astrophysics and Space Science Library, p. 45

[15] S.R. Kulkarni, E.O. Ofek, J.D. Neill, Z. Zheng, M. Juric, The Astrophysical Journal 797, 70 (2014), 1402.4766 